DOI: 10.30972/eitt.704781

\title{
Análisis de la labor ingenieril en tiempos de COVID-19
}

Alicia I. Amarilla, Norberto A. Sanabria, Alicia S. Cuevas( ${ }^{*}$ )

\section{Resumen}

Este trabajo tiene como objetivo indagar sobre el efecto de la pandemia provocada por el COVID-19, sobre el ejercicio de la ingeniería, tanto en el ejercicio profesional independiente, como en las empresas conducidas por estos profesionales egresados de la Universidad Nacional del Nordeste (UNNE); explorando además sobre su capacidad de adaptación a las nuevas demandas del mercado laboral.

Para el desarrollo del presente escrito se contó con la colaboración de profesionales del medio y alumnos del último ciclo de las carreras de ingeniería eléctrica e ingeniería en electrónica (especialidades biomédica y telecomunicaciones), quienes conforman la población analizada.

\section{Introducción:}

La situación actual que enfrenta el mundo ante la pandemia del COVID-19, ha impactado directamente en el ejercicio de muchas profesiones y empresas, que han sido afectados en sus procesos productivos de bienes y servicios. En este contexto, 
la ingeniería juega un papel muy importante al momento de replantear estrategias y explorar soluciones que permitan dar continuidad a las empresas de la zona del Nordeste Argentino (NEA).

En esta situación es importante entender que las carreras de ingeniería forman a los profesionales con conocimientos específicos según su disciplina, y capacidad para trabajar en la planificación, composición, evaluación, asesoramiento, dictamen, directiva, supervisión o gerenciamiento de proyectos.

Desde el inicio de las restricciones de circulación y el confinamiento debido al coronavirus, se destacan dos situaciones presentadas: en la primera, muchas empresas debieron suspender total o parcialmente sus actividades laborales, lo cual afectó significativamente su productividad; en la segunda, las empresas pudieron continuar con su actividad productiva esencial, pero no contaban con los recursos necesarios para atender a la demanda creciente de sus clientes, tanto de bienes como de servicios de ingeniería. En este estudio nos enfocamos en las disciplinas eléctrica y electrónica (biomédica y telecomunicaciones). Por ello debieron readecuar su estructura y funcionamiento.

Palabras Clave: trabajo, organización, ingeniería, COVID-19.

\section{Marco Teórico:}

El desarrollo de toda actividad laboral, en función de las condiciones y medio ambiente de trabajo, establece una relación directa y permanente con la vida y la salud de todo trabajador, motivo por el cual se considera un elemento clave en los distintos contextos que demanda la sociedad.

El trabajo permite o contribuye a la realización individual, es decir al desarrollo de la personalidad de quienes lo ejecutan y define una identidad social cristalizada en la profesión o el oficio. Durante su ejercicio el trabajo permite la actualización de todas, o al menos algunas, de las capacidades físicas, biológicas, psíquicas y mentales del ser humano, condicionadas por los sentimientos de pertenencia o inserción 
en un grupo, categoría o profesión. Asimismo, el trabajo construye la identidad de las personas, algo que distingue a cada uno respeto de los otros trabajadores, pero que dependen de la mirada, la crítica, el apoyo y la evaluación de los otros. El cuestionamiento de la pertenencia de un trabajador a un colectivo social y de su identidad productiva, son fuentes de preocupación y angustia. Neffa, 2015.

su vez, el trabajar significa siempre asumir riesgos pues, para cumplir los objetivos, la actividad que efectivamente desarrolla la persona se diferencia del trabajo prescripto por quienes dirigen o gestionan el uso de la fuerza laboral. También implica una movilización de los recursos psíquicos y mentales del trabajador pues para generar el bien o el servicio requiere de su motivación e involucramiento, tanto en el nivel individual como en el colectivo: necesita captar, interpretar y procesar la información, comunicarse, expresar sus ideas por medio de la palabra y mediante silencios, gestos y signos; promueve coordinación y cooperación dentro del grupo de trabajo; evalúa el resultado de su esfuerzo. Para llevarse a cabo eficazmente requiere no sólo un compromiso físico, sino también subjetivo, es decir psíquico y mental con la tarea desarrollada.

La importancia de analizar el trabajo en la vida del ser humano es porque su labor socializa a las personas y es fuente de inserción social. Se establecen relaciones interpersonales que pueden ser de amistad, de apoyo, de comunicación y de cooperación, pero también conflictivas y de competencia. La organización de tareas cumple un papel determinante, ya que no surge repentinamente sino que se construye como un proceso continuo.

\section{Organizaciones:}

La vida de los individuos está conformada por muchas interacciones con otras personas y con organizaciones. El ser humano es eminentemente social e interactivo, no vive aislado sino en convivencia y en relación constante con otros. Una organización es un sistema abierto de actividades conscientemente coordinadas de dos o más personas.

En la sociedad moderna, casi todo proceso productivo se realiza por medio de las organizaciones: instituciones, empresas, organismos. El hombre moderno pasa 
la mayor parte de su tiempo en las organizaciones, de las que depende para nacer, vivir, aprender, trabajar, ganar su salario, curar sus enfermedades, obtener todos los productos y servicios que necesita. Chiavenato, 2007.

Desde un punto de vista más amplio, las organizaciones son unidades sociales o agrupaciones humanas, intencionalmente constituidas y reconstruidas para el logro de objetivos específicos. Esto significa que se fundan de manera planeada y organizada para el logro de objetivos determinados. A medida que se logran los objetivos y que se descubren medios para obtenerlos a menor costo y con menor esfuerzo, las organizaciones se reconstruyen, es decir, se reestructuran y se redefinen. Una organización no es nunca una unidad completa y terminada, sino un organismo social vivo y sujeto a constantes cambios. A la vez, todo sistema opera sobre la materia, la energía o la información obtenidas del ambiente, las cuales constituyen los insumos o entradas (inputs) de recursos necesarios para que el sistema pueda operar. Esos recursos son operados por las diversas partes del sistema (subsistemas) y transformados en salidas o resultados (outputs) para ser devueltos al ambiente. Pero además de los recursos, las organizaciones necesitan de competencias.

Según Gili, la estructura es la disposición de las partes adecuada a los objetivos, que comprende su agrupamiento y el análisis de sus relaciones. La estructura formal está determinada por el conjunto de posiciones oficiales que integran el sistema. Las organizaciones utilizan el organigrama para representar gráficamente la estructura formal, la división de las tareas y las jerarquías de las diferentes posiciones. Las prescripciones no contemplan las expectativas de los individuos. Aun suponiendo que los miembros de una organización perciban adecuadamente lo que se espera de ellos, es posible que las prescripciones no se cumplan por falta de motivación o mala coordinación de las actividades. La estructura real es la suma de la estructura formal y la informal. Con "informal" nos referimos a las relaciones no oficiales que complementan y, a veces, reemplazan a las prescriptas. Algunos autores definen cuatro tipos de estructuras organizacionales, como más típicas.

La estructura de la organización es "el conjunto de todas las formas en que se divide el trabajo en tareas distintas, consiguiendo luego la coordinación de las mismas” (Mintzberg, 1989). La definición comprende dos aspectos: por un lado, cómo 
diferenciar las tareas, y por el otro, cómo integrarlas, mediante la coordinación, para alcanzar los objetivos definidos previamente. La diferenciación y la integración son elementos claves de la estructura organizativa.

Considerando la definición de las "estructuras de red", las cuales se dan ante las nuevas realidades de los mercados regionalizados, las organizaciones zonales podrían buscar nuevas formas estructurales e interorganizacionales. Las redes organizacionales parecen ser una adecuada respuesta frente a los altos costos provenientes del desarrollo de productos o servicios y la necesidad de penetrar en mercados más exigentes.

Las estructuras de red se están ampliando, en parte por el desarrollo de los sistemas de información y comunicación, que hacen más fácil la coordinación entre proveedores y clientes. Estas surgen para hacer frente a la fuerte competencia y al rápido cambio tecnológico, y consiste en la descentralización vertical, a partir de la delegación de la producción de variados productos y servicios, en otras organizaciones; las redes son en sí mismas empresas industriales sin producción final, y pueden llegar a ser un modelo adaptable a la era posindustrial. Además de la búsqueda de fuentes externas de productos terminados, aparecen otras formas de asociación, tales como joint-ventures y alianzas temporarias. Las empresas se repliegan hacia sus actividades esenciales, eliminan niveles jerárquicos y terciarizan un amplio rango de funciones. Existen cada vez más ejemplos de organizaciones que adoptan la forma de redes. Las estructuras en red no sólo incluyen a las grandes empresas u organizaciones, sino también a las pequeñas y medianas empresas (PyME), ya que algunas de las formas asociativas consisten en la diversificación de una gran empresa mediante el establecimiento de vínculos comerciales y de asistencia con una PyME, con una relación de colaboración entre colegas. Gilli, 2007.

\section{Análisis y Resultados:}

Habiendo definido e identificado algunos conceptos básicos, a continuación se exponen los datos necesarios para analizar el contexto en el cual se enmarca el trabajo de investigación y el área en el cual desarrollan su labor la población de estudio: alumnos del ciclo profesional y graduados de las carreras de Ingeniería Eléctrica y Ingeniería en Electrónica. 
En el contexto socioeconómico del NEA, según datos del Instituto Nacional de Estadística y Censos de la Argentina (INDEC, 2020) las tasas de la población con nivel educativo superior y universitario completo, respecto a la Población Económicamente Activa (PEA), son significativas. Los aglomerados o ciudades de la región NEA tienen estas tasas con los valores siguientes: Formosa: 18,2 \%; Gran Resistencia: 16,7\%; Corrientes: 26,5\%; Posadas: 27,1\%.

Si bien el primer trimestre del año 2020 registró valores de desocupación del $5,4 \%$ y la tasa de demanda de empleo fue del 21,2\%; a partir de las restricciones de movilidad y la crisis económica generada por la pandemia del COVID-19, estos valores crecieron en todos los rubros, incluyendo las disciplinas eléctrica y electrónica.

A nivel nacional para el segundo trimestre del año 2020, la tasa de desocupación subió al 13,1\%, con un incremento de 2,5\% interanual. Mientras tanto, la desocupación demandante bajó del 9,2\% al 5\% con el confinamiento, y la desocupación no demandante creció del 3,9\% hasta ubicarse en el 4,6\%.

En un contexto cambiante y con restricciones sociales, económicas, laborales, tecnológicas, científicas y educativas, la ingeniería como profesión ligada al desarrollo de un país toma mayor relevancia. Entre otros aspectos, la ingeniería por su participación activa en los procesos de innovación e implementación de los avances en ciencia y tecnología, tomó un rol esencial en la adecuación al nuevo escenario.

En el segundo trimestre de 2020, coincidente con el inicio de las medidas de emergencia sanitaria, la región NEA tuvo un abrupto crecimiento de desocupación y caída del empleo demandado, en un contexto fuertemente marcado por la pandemia del COVID-19. Comparado con otras regiones de la Argentina, el NEA tuvo la segunda tasa más baja del país en cuanto a niveles de actividad: $37,1 \%$.

Analizando los conceptos en situación de pandemia, tenemos por otro lado que las instituciones gubernamentales, como las asociaciones profesionales de la región coinciden en afirmar que existe una importante demanda insatisfecha para contar con ingenieros en sus planteles. Las pequeñas y medianas empresas (PyME) y los 
emprendedores también buscan la asistencia de ingenieros preparados para relacionarse con el mundo de los negocios; este es un perfil altanamente demandado actualmente.

\section{Aspectos Metodológicos:}

Para la producción de conocimientos y responder a los objetivos complejos que se plantean, se utiliza la mezcla de métodos (Mixed-Method). Ésta se define como la combinación de una aproximación cuantitativa y una cualitativa dentro de un mismo proceso de investigación con el objeto de obtener una comprensión más profunda sobre los problemas considerados relevantes, aumentando la confianza en los datos, la captación de procesos y correlaciones, y disponer de un contexto social que enmarque la acción (Fielding, 2012).

La población de estudio está compuesta por graduados y alumnos del ciclo profesional de las carreras de Ingeniería Eléctrica e Ingeniería en Electrónica de UNNE, que tienen o participan de organizaciones formales y que ofrecen bienes y servicios en la región del NEA.

La unidad de análisis utilizada para esta instancia es de 25 personas, de las cuales 10 son alumnos de ingeniería y 15 son graduados, todos ellos trabajan en organizaciones privadas o son dueños de su propia empresa o emprendimiento.

Las técnicas de recolección de información utilizadas fueron entrevistas semi estructuradas abiertas del tipo sincrónica y asincrónica; lectura y análisis de documentos y de los datos cuantitativos y cualitativos obtenidos.

Para el análisis se tuvo en cuenta a aquellas empresas ubicadas geográficamente en el NEA. De las seleccionadas el 60\% se encuentra en Corrientes, el 20\% en Resistencia y el 10\% con alguna sucursal en Misiones.

Analizando el contexto socioeconómico según informes del INDEC y los datos cuantitativos y cualitativos del relevamiento propio, se puede afirmar de los profesionales que desarrollaban en forma independiente su actividad ha aumentado en $50 \%$ su producción laboral, a partir de la liberación paulatina del aislamiento pre- 
ventivo, al igual que las empresas tuvieron que incorporar personal con conocimientos técnicos debido al aumento de la demanda de servicios y productos derivados de las ingenierías.

Otro factor relevante que se detectó a partir de las entrevistas es que las grandes empresas debieron contratar a empresas más pequeñas para dar respuestas más rápidas a la alta demanda del mercado. En el área de telecomunicaciones para instalación y mantenimiento de antenas de telefonía móvil, de internet domiciliario, entre otras; en la especialidad eléctrica, con el relevamiento, desarrollo, mantenimiento y puesta en funcionamiento de instalaciones eléctricas, principalmente en baja tensión: acometida, protecciones, distribución, alimentación e iluminación, como así también refrigeración; y para la especialidad biomédica en el mantenimiento, instalación y puesta en marcha de equipos biomédicos, adecuación de recintos. En el área electrónica también hubo una creciente solicitud de servicios de vigilancia y monitoreo a través de la instalación de cámaras y sensores de movimiento. La demanda de estos servicios de ingeniería alcanzó un aumento de hasta un 60\% promedio respecto al 2019 .

Los hallazgos obtenidos se organizaron en dos apartados principales. El primero, presenta datos derivados de un análisis cuantitativo referente a las características de la trayectoria de las empresas de la población de estudio y por otro, las consecuencias y adaptación de su actividad en el contexto de pandemia COVID-19. Esta adecuación se vió reflejada en la reestructuración de sus organigramas en muchos casos y en la organización y distribución de la fuerza laboral.

Como lo señalan los datos, se advierte un aumento de la inserción laboral de los graduados y de los alumnos del ciclo superior en Ingeniería Eléctrica e Ingeniería en Electrónica, en el contexto sanitario nombrado, indicando una alta empleabilidad en sus diversas modalidades y en la inserción en empresas.

Más del 50\% de los alumnos entrevistados afirmaron que en virtud del aumento de la demanda de servicios de ingeniería fueron convocados a través de personas conocidas y de profesionales del medio, a incorporarse en empresas ya constituidas formalmente para la realización de tareas específicas de las disciplinas analizadas. 
A su vez, profesionales independientes conformaron equipos de trabajo debido a la necesidad de dar soluciones rápidas a los requerimientos de los clientes.

\section{Conclusiones:}

En este trabajo buscamos conocer la situación de las empresas del medio dedicadas al servicio y producción de bienes, derivados de la ingeniería y en el ejercicio de la profesión en un contexto restricciones y confinamientos parciales por Coronavirus. Del mismo modo indagamos sobre el mercado laboral de los alumnos del ciclo superior y profesionales independientes de las carreras de Ingeniería Eléctrica y en Electrónica de la UNNE.

Los resultados obtenidos revelaron una situación que se enmarca, en una mayor demanda de servicios de ingeniería, tanto para los profesionales, las empresas conformadas por ellos y los alumnos avanzados, especificando el contexto y la situación económica del país y del mundo, como consecuencia del COVID-19.

Esta situación de pandemia favoreció la socialización entre personas de formación similar. Se refleja mayor comunicación y coordinación de tareas, en primer lugar entre todos los que trabajan en las empresas: socio-profesionales de mayor nivel jerárquico de una misma unidad económica y establece directa o indirectamente, una "relación de servicio" con los clientes, usuarios y los trabajadores. Asimismo, se repite entre los profesionales independientes, al conformar "equipos" para llevar adelante los trabajos requeridos.

Tanto para los profesionales independientes, como para aquellos que poseen un emprendimiento o empresas, constituyó un desafío importante la necesidad de adecuarse a la nueva situación, debiendo readecuar no sólo la producción de bienes y servicios, si no también realizar algunas modificaciones sustanciales como la reingeniería de sus estructuras orgánicas y funcionales, encontrando en las "estructuras en red y de equipos" una innovación, las cuales se aplican ante las nuevas realidades de los mercados regionalizados o las organizaciones zonales, y buscar nuevas formas estructurales e interorganizacionales. Las redes en las organizaciones parecen ser una adecuada respuesta frente a los altos costos provenientes del desarrollo de productos o nuevos servicios y la oportunidad de entender mercados más exigentes 
y específicos en el escenario actual. Asimismo, el uso más frecuente de sistemas de información y comunicación, hace más fácil la coordinación entre proveedores y clientes, dando lugar a un mayor impacto y desarrollo para las ingenierías.

\section{Referencias:}

- Chiavenato I. “Administración de Recursos Humanos. El capital humano de las organizaciones”. Octava edición. Editorial McGraw-Hill / Interamericana Editores SA. ISBN 970-10-6104-7. México. Año 2007.

- Gilli J.J. et al. "Diseño organizativo. Estructura y Procesos”. Editorial Granica SA. ISBN 978-950-641-514-3. Buenos Aires, Argentina. Año 2007.

- Góngora N. et al, "La cultura organizacional y el trabajo en equipo”. Instituto de Investigaciones Administrativas de la FCE de la UNLP. La Plata. Argentina. Año 2019.

- Neffa, J.C. "Los Riesgos Psicosociales en el Trabajo. Contribución a su estudio". Libro Digital ISBN 978-987-21579-9-9. Año 2015.

- Nieto, H. "Epidemiología de los accidentes de trabajo entre los trabajadores sanitarios”. Rev. del Instituto de Higiene y Med. Social. Año 1999.

- Rodríguez Gómez, G. "Metodología de la Investigación Cualitativa”. Editorial Alsives. Málaga, España. Año 1999.

- Sabino, C. "El proceso de Investigación”. Editorial Hvmanitas. Buenos Aires, Argentina. Año 1996.

- Vasilachis de Gialdino, I. "Estrategia de Investigación Cualitativa. Editorial Gedisa. Buenos Aires, Argentina. Año 2007.

- Verón, O.E. “Lineamientos Estratégicos”. Superintendencia de Riesgos de Trabajo. Ed. SRT. Buenos Aires, Argentina. Año 2003.

- Vicente M.A. y Ayala J.C. "Principios fundamentales para la administración de organizaciones”. Editorial Prentice Hall - Pearson Educación. ISBN 9789876150156. Buenos Aires, Argentina. Año 2008.

- Villate, R. "El método del árbol de causas: para analizar los accidentes de trabajo en vistas a su prevención”. Ed. Área de Estudios e Investigaciones Laborales de la SECYT, CEIL-CONICET, CREDAL-CNRS. Hvmanitas. Año 1990.

- Volkoff, S. "Estadísticas sobre condiciones y medio ambiente de trabajo. Méto- 
dos y resultados”. Editorial Asociación Trabajo y Sociedad. Buenos Aires, Argentina. Año 1993.

- INDEC "Mercado de trabajo. Tasas e indicadores socioeconómicos (EPH) Segundo trimestre de 2020, Informes técnicos”. Vol. 4, nº 232 ISSN 2545-6636. Buenos Aires, Argentina. Año 2000. 